\title{
Spatial and Frequency Compounding in Application to Attenuation Estimation in Tissue
}

\author{
Ziemowit KLIMONDA, Jerzy LITNIEWSKI, Piotr KARWAT, Andrzej NOWICKI \\ Institute of Fundamental Technological Research \\ Polish Academy of Sciences \\ Pawińskiego 5B, 02-106 Warszawa, Poland; e-mail: \{zklim, jlitn, pkarwat, anowicki\}@ippt.pan.pl
}

(received March 10, 2014; accepted September 5, 2014)

\begin{abstract}
The soft tissue attenuation is an interesting parameter from medical point of view, because the value of attenuation coefficient is often related to the state of the tissue. Thus, the imaging of the attenuation coefficient distribution within the tissue could be a useful tool for ultrasonic medical diagnosis. The method of attenuation estimation based on tracking of the mean frequency changes in a backscattered signal is presented in this paper. The attenuation estimates are characterized by high variance due to stochastic character of the backscattered ultrasonic signal and some special methods must be added to data processing to improve the resulting images. The following paper presents the application of Spatial Compounding (SC), Frequency Compounding (FC) and the combination of both. The resulting parametric images are compared by means of root-mean-square errors. The results show that combined SC and FC techniques significantly improve the quality and accuracy of parametric images of attenuation distribution.
\end{abstract}

Keywords: tissue attenuation estimation, parametric imaging, synthetic aperture, spatial compounding, frequency compounding.

\section{Introduction}

The standard ultrasonic images - the brightness mode or B-mode images - present the distribution of the tissue reflectivity which is related mostly to the tissue acoustic impedance variation. This is a well known modality and is widely used in medical diagnostics. However, there are some other tissue parameters such as backscattering coefficient, attenuation coefficient and speed of sound which are strictly related to the tissue structure and could provide additional diagnostic value. The attenuation coefficient is a matter of interests due to potentially substantial importance in medical diagnostic. The attenuation properties of pathological tissue often differ significantly from the healthy tissue. For example, the attenuation can vary from several percent for cirrhotic human liver, through dozens percent for fatty human liver (LU et al., 1999). Similar changes were noted for degenerated bovine articular cartilage (NIEMINEN et al., 2004). The reported attenuation value increases over a hundred percent in case of porcine liver HIFU treatment in vivo (ZDERIC et al., 2004) or two hundred percent for porcine kidney thermal coagulation (WoRTHINGTON, SHERAR, 2001). It has been also reported that the attenuation coefficient differs for cancerous and healthy tissue (SAIJO, SASAKI, 1996). Moreover, the noninvasive determination of ultrasonic attenuation enables to predict the premature delivery in rats and humans (BIGELOW et al., 2008; MCFARLin et al., 2010). These reports motivate us to search for efficient methods of unambiguous attenuation estimation to be used in ultrasonic (US) imaging. The approach used in following paper bases on tracking of the variation of the mean frequency of the echoes along reconstructed image lines. However, earlier examinations showed large variability of the attenuation coefficient estimated through this method (KLimonda et al., 2009). Such variability introduces ambiguity and limits the resolution and accuracy of parametric images. It can be reduced by averaging of adjacent image lines at a cost of reduced lateral resolution. The effectiveness of averaging depends on the number of averaged lines and their statistical independence which is inversely related to the acoustic beam width. The increase of the statistical independence of the image lines can be achieved through Synthetic Aperture Focusing Technique (SAFT) which, unlike the Classical Beamforming ( $\mathrm{CB})$, provides high image resolution in the whole scanned area. Some results of this approach 
can be found in our previous work (KLIMONDA et al., 2011). Another approach is to improve the attenuation estimates by means of compounding techniques. The number of the image lines can be increased with the use of the Spatial Compounding (SC) technique. Additionally, the variance of the attenuation maps can be reduced by means of Frequency Compounding (FC) technique. Therefore, all of above-mentioned methods were used for the collection of the acoustic data which were subsequently processed for the attenuation estimation. The improvement of the quality of attenuation images for four approaches (CB, CB combined with FC, CB combined with SC, CB combined with both $\mathrm{FC}$ and $\mathrm{SC}$ ) was assessed and compared.

\section{Theory}

\subsection{Estimation of attenuation coefficient}

The ultrasonic wave propagating through a soft tissue is attenuated due to absorption and scattering. The amplitude $A$ of the plane wave decreases exponentially with the propagation distance, which can be expressed as

$$
A(x)=A_{0} \exp (-\alpha(f) \cdot x)
$$

where $A_{0}$ is an initial amplitude, $\alpha(f)$ is a frequency dependent acoustic pressure attenuation coefficient and $x$ is a distance passed by the wave within the tissue. The attenuation coefficient is the sum of the scattering and absorption coefficients. However, for the frequencies used in standard ultrasound imaging (1$15 \mathrm{MHz}$ ) most of the attenuation in the soft tissue is caused by absorption. In the soft tissue the frequency dependent attenuation coefficient can be approximated by the following empirical expression (COBBOLD, 2007)

$$
\alpha(f)=\alpha_{1} f^{n}
$$

where $\alpha_{1}$ denotes the attenuation coefficient at the frequency equal to $1 \mathrm{MHz}$ and $n$ is a positive exponent. The value of $n$ is typically close to 1 for the soft tissues and it is common to assume $n=1$, which means that the attenuation increases linearly with frequency. Thus, according to (2) higher frequency components of a propagating pulse are attenuated more strongly than lower frequency components. Let us assume that the pulse has a Gaussian envelope in time domain and is given by the equation:

$$
p(t)=a_{0} \cos \left(2 \pi f_{0} t\right) \exp \left(-2 \pi^{2} \sigma_{0}^{2} t^{2}\right),
$$

where $a_{0}, f_{0}$ and $\sigma_{0}^{2}$ is the pulse amplitude, carrier frequency and spectral variance, respectively. Thus, its spectrum obtained via Fourier transform is given by the following equation:

$$
P(f)=\frac{a_{0}}{2 \sigma_{0} \sqrt{2 \pi}} \exp \left(-\frac{\left(f-f_{0}\right)^{2}}{2 \sigma_{0}^{2}}\right) .
$$

The power spectrum $R(f, x)$ of a Gaussian shaped pulse propagating through attenuating medium can be described in frequency domain based on Eqs. (1), (2) and (4) by the equation:

$R(f, x)=\left(\frac{a_{0}}{2 \sigma_{0} \sqrt{2 \pi}}\right)^{2} \exp \left(-\frac{\left(f-f_{0}\right)^{2}}{\sigma_{0}^{2}}\right) \exp \left(-2 \alpha_{1} f x\right)$.

The second exponential term represents the attenuation. Equation (5) can be transformed to the following form

$$
\begin{aligned}
R(f, x)= & \left(\frac{a_{0}}{2 \sigma_{0} \sqrt{2 \pi}}\right)^{2} \exp \left(-\frac{2 \sigma_{0}^{2} \alpha_{1} x f_{0}-\left(\sigma_{0}^{2} \alpha_{1} x\right)^{2}}{\sigma_{0}^{2}}\right) \\
& \cdot \exp \left(-\frac{\left(f-\left(f_{0}-\alpha_{1} x \sigma_{0}^{2}\right)\right)^{2}}{\sigma_{0}^{2}}\right)
\end{aligned}
$$

The product of square term and first exponential term in Eq. (6) is not dependent on $f$ and represents the maximal value of the pulse power spectrum. The second exponential term represents the Gaussian spectrum with mean frequency described by Eq. (7) (LAUGier et al., 1985; SzABo, 2004).

$$
f_{m}=f_{0}-\alpha_{1} \cdot x \cdot \sigma_{0}^{2} .
$$

Moreover, formula (6) shows that the Gaussian pulse preserves its spectral shape during propagation in linearly attenuating medium i.e. the $\sigma_{0}^{2}$ is constant. Therefore, when a pulse propagates through attenuating medium, a shift of its mean frequency towards lower frequencies is observed, which is shown in the Fig. 1. Thus, the attenuation coefficient can be calculated according to the equation:

$$
\alpha_{1}=-\frac{1}{\sigma_{0}^{2}} \frac{\Delta f_{m}}{\Delta x} \underset{\Delta x \rightarrow 0}{\longrightarrow}-\frac{1}{\sigma_{0}^{2}} \frac{d f_{m}}{d x},
$$

where $\Delta f_{m}=f_{m}-f_{0}$ is a change of the mean frequency when the pulse passes the $\Delta x$ distance.

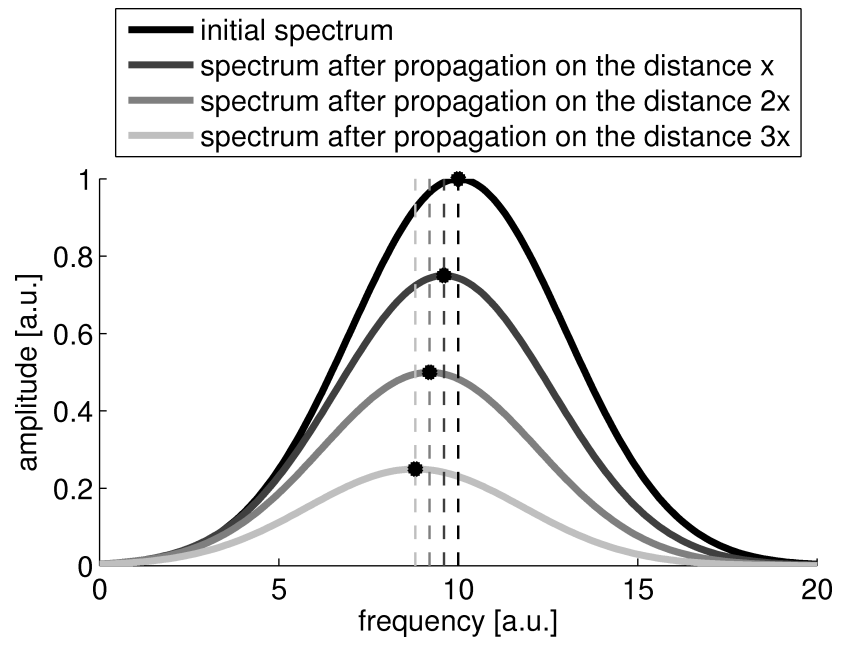

Fig. 1. Simulation of the spectrum of the Gaussian pulse propagating in linearly attenuating medium. The mean frequencies of the spectra are represented by dashed lines.

Their shifts toward the lower frequencies are visible. 


\subsection{Spatial and Frequency Compounding}

The attenuation maps obtained by direct use of Eq. (8) are characterized by very high variance which affects the quality and accuracy of the parametric image. The attenuation image quality can be improved by means of spatial compounding (SC) technique at the cost of decrease of the frame-rate. This technique involves "looking" at the examined object at different angles which can be achieved electronically - by applying proper time delays to the transmitting transducers, or mechanically - by tilting the probe (Fig. 2). In this study the SC was performed mechanically. The final image is an average of images obtained from several different, closely located scan planes. Such averaging significantly reduces the variation in the final attenuation map (KLIMONDA et al., 2010) Additionally, the frequency compounding (FC) technique can be used. The FC technique involves filtration of each RF line into several narrow frequency band RF lines In frequency domain it corresponds to the partition of the RF line spectrum into several narrow frequency bands (see Fig. 3). These filtrated narrow-band signals are processed separately to obtain the mean frequency es-

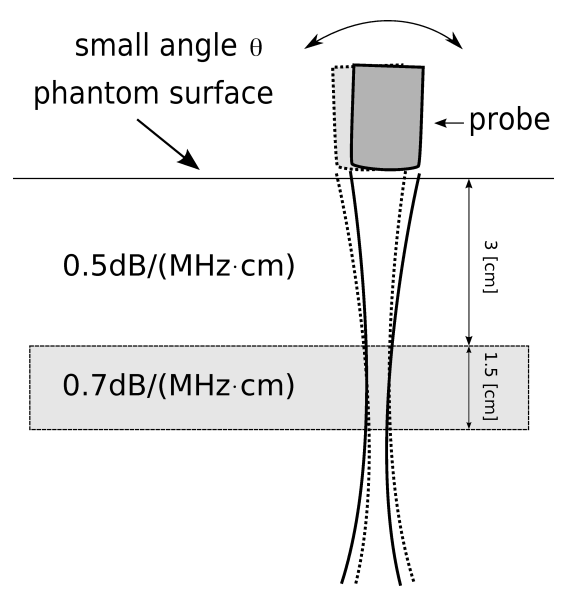

Fig. 2. The idea of SC technique used in this study. The successive imaging planes are produced by tilting the probe.

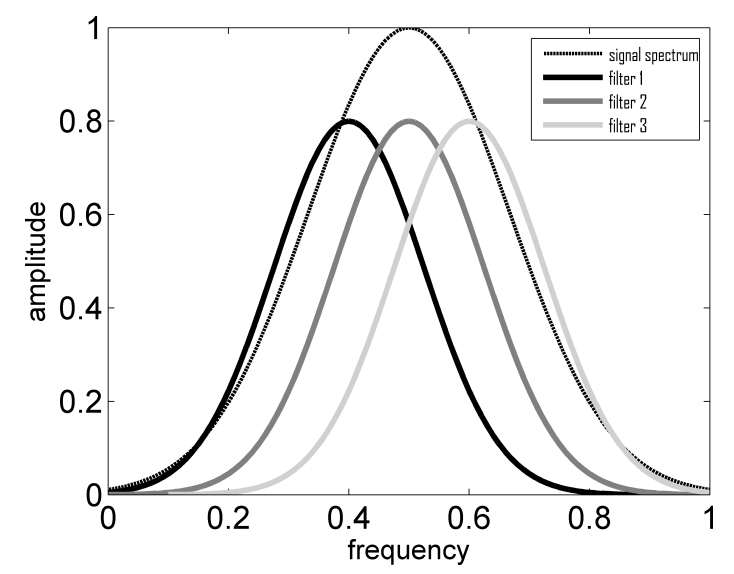

Fig. 3. The idea of the partition of the echo-line spectrum used in FC technique. Only three filters are presented while typically the number of filters is much larger. timates which next undergo the energy-weighted averaging. Then, the averaged mean frequency estimate is processed according to Eq. (8) to obtain the final attenuation map.

\section{Measurements and signal processing}

\subsection{Materials}

Experimental data was acquired from two tissue mimicking phantoms (1126 A and B, Dansk Phantom Service, Denmark) with uniform echogenicity and attenuation coefficient equal to $0.5 \mathrm{~dB} /(\mathrm{MHz} \cdot \mathrm{cm})$. The first phantom (1126 A) additionally contained a cylinder of $15 \mathrm{~mm}$ in diameter located at $30 \mathrm{~mm}$ depth. Regarding the echogenicity, the cylinder was identical to the surrounding medium. However, it had different attenuation coefficient value equal to $0.7 \mathrm{~dB} /(\mathrm{MHz} \cdot \mathrm{cm})$. The B-mode image from the phantom is presented in Fig. 4. The cylinder is almost invisible because of uniform echogenicity so it was marked by white circle. The presence of the phantom is manifested by two specular echoes from borders at the top and the bottom of the cylinder. Moreover, the acoustical shadow located beneath the cylinder is also visible. The second phantom (1126 B) was used as a reference phantom enabling correction of effects related to diffraction and decreasing signal-to-noise ratio (SNR).

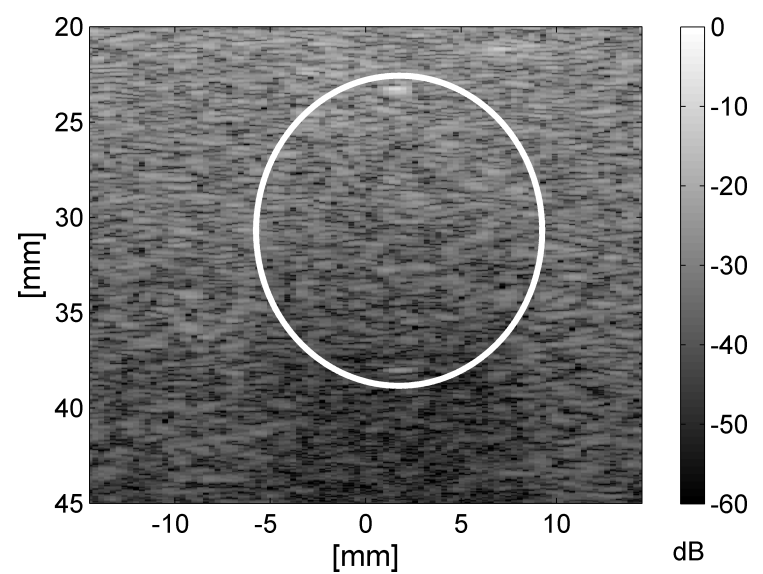

Fig. 4. The B-mode image of high attenuation cylinder in the tissue phantom.

The ultrasonic data was collected using the SonixTOUCH scanner (Ultrasonix, Canada) with linear probe L14-5/38 and next processed off-line using Matlab ${ }^{\circledR}$. The scanner allowed to record raw prebeamformed data at sampling frequency of $40 \mathrm{MHz}$. The focal point was located at $3 \mathrm{~cm}$ depth. Short wideband pulses of $8 \mathrm{MHz}$ center frequency were used as an excitation.

\subsection{Mean frequency estimation}

In our approach the mean frequency $f_{m}$ is directly evaluated from the backscattered signal along 
the propagation path by means of the correlation estimator. The estimator is described by Eq. (9) (EvAns, MCDICKEN, 2000)

$$
\begin{aligned}
f_{m} & =\frac{1}{2 \pi T_{s}} \\
\cdot \operatorname{atan} & \left(\frac{\sum_{i=1}^{N} Q(t(i)) I\left(t(i)+T_{s}\right)-Q\left(t(i)+T_{s}\right) I(t(i))}{\sum_{i=1}^{N} I(t(i)) I\left(t(i)+T_{s}\right)+Q\left(t(i)+T_{s}\right) Q(t(i))}\right),
\end{aligned}
$$

where $t$ is the time, $T_{s}$ is the sampling period and $N$ is the estimator window length. The $Q$ and $I$ are quadrature and in-phase signal components that are obtained with quadrature sampling technique. The signal samples are numbered with index $i$. The quadrature sampling is often used in modern scanners while the correlation estimator is widely used in color Doppler imaging (Evans, MCDiCKEn, 2000).

The received $\mathrm{RF}$ echo signals were reconstructed into RF image lines which were next analyzed for mean frequency changes by means of correlation estimator. The single RF line passing through the cylinder and corresponding $f_{m}$ line is presented in Fig. 5. The vari-

a)

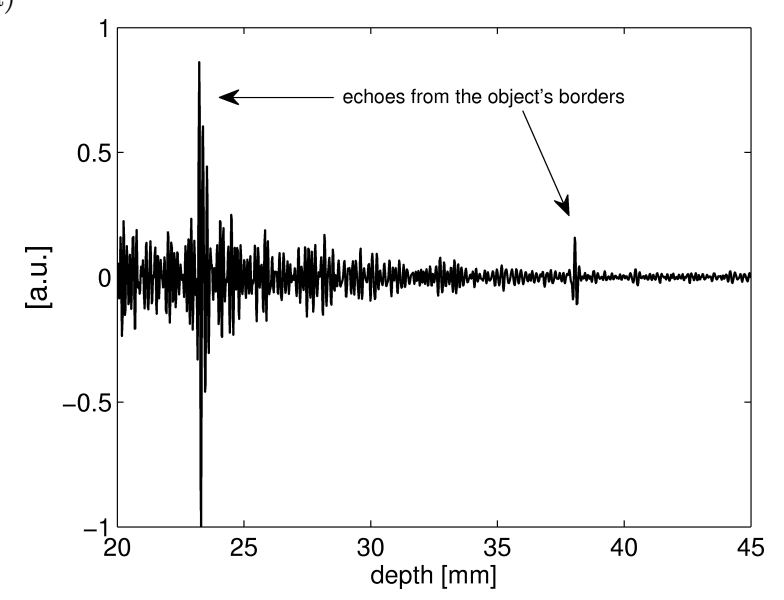

b)

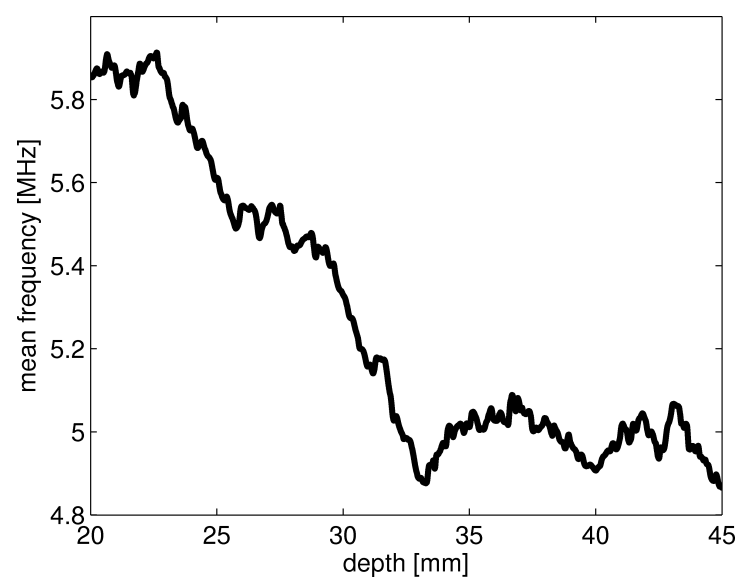

Fig. 5. The RF line corresponding to echoes from the attenuating cylinder (a) and corresponding $f_{m}$ line (b). ation of the $f_{m}$ line reflects the random character of the backscatter signal and forces us to apply the averaging and trend extraction techniques as well as spatial and frequency compounding.

In order to lower the variance of the resulting $f_{m}$ lines, additional moving average filtration is performed laterally. The moving average window length generally corresponds with the depth covered by $N$ samples window of correlation estimator. The resulting $f_{m}$ line is next processed by Singular Spectrum Analysis (SSA) method (GolyAndina et al., 2001) to obtain smooth, decreasing trend (see Fig. 6). The SSA technique decomposes the input data series into the sum of components which can be interpreted as a trend, oscillatory components and noise (non-oscillatory components). The major applications of the SSA technique are smoothing of time series, finding trends, forecasting and detecting structural changes. There are two interesting features of SSA in the context of the determination of the ultrasound attenuation profiles using signal's mean frequency changes. Firstly, this is a model-free technique which means that there is no need to know general function describing how the mean frequency changes with the depth. In fact, in a clinical situation there is no a priori knowledge about it. All we know is that the mean frequency changes should be a strictly decreasing function along the path length. Furthermore, the local value of the attenuation coefficient should be in a certain range which depends on a tissue type. The second feature of the SSA is the robustness to outliers. This is an important advantage as outliers could appear in the depth dependence of the mean frequency of the real radio-frequency ( $\mathrm{RF}$ ) echoes acquired from tissue. Moreover the SSA is easy to use as it needs only one parameter - the window length. The mean frequency trend obtained with the SSA was

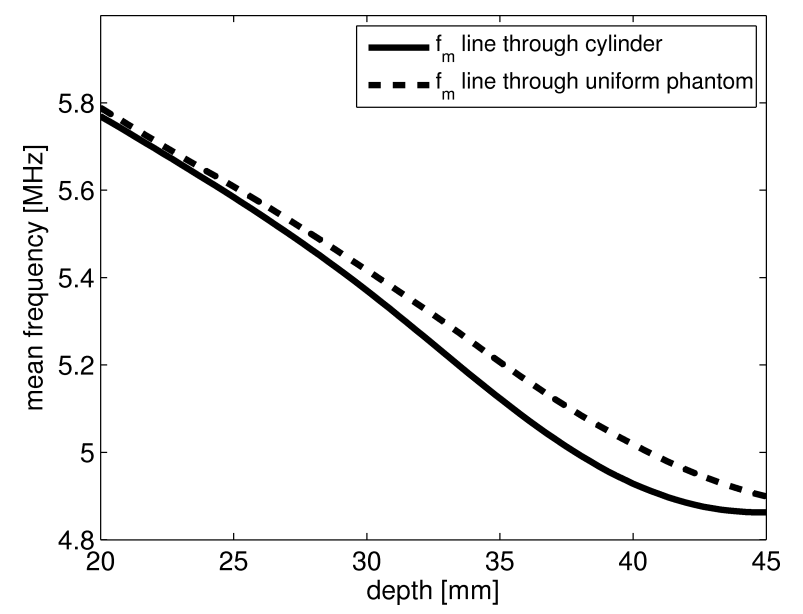

Fig. 6. The $f_{m}$ lines calculated for echoes scattered in high attenuating cylinder and uniform phantom. The $f_{m}$ lines were averaged and processed by SSA.The increased drop of $f_{m}$ estimate due to propagating through the cylinder is visible. 
next used in calculation of the attenuation along the propagation path according to Eq. (8). All processing techniques: mean frequency estimation, moving average filtration and the SSA used the $10 \mathrm{~mm}$ length windows. The example of averaged $f_{m}$ lines processed by means of SSA algorithm is presented in Fig. 6 . The dashed $f_{m}$ line comes from uniform phantom, the solid comes from phantom with the high cylinder of increased attenuation coefficient. The effect of cylinder attenuation is visible - the solid $f_{m}$ line decreases faster.

\section{3. $S C$ and $F C$ processing}

The spatial compounding (SC) was done mechanically by tilting the probe in $\pm 10^{\circ}$ range around the array-phantom contact line (Fig. 2). The resulting geometrical distortions were small in comparison to the resolving power of the attenuation imaging technique,

a)

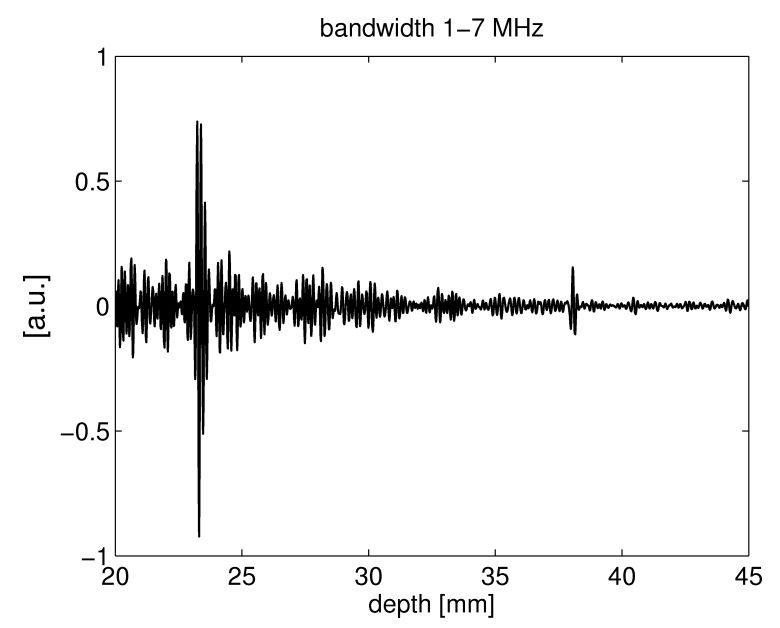

b)

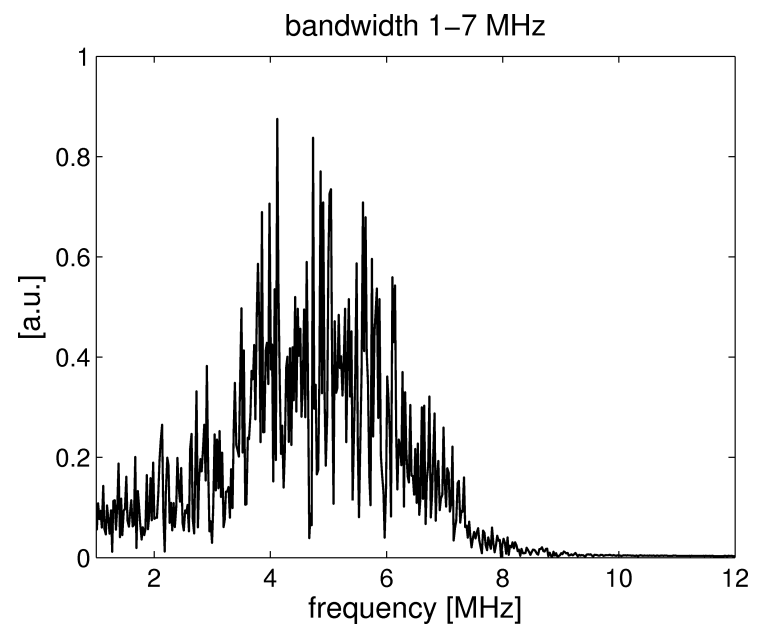

Fig. 7. The filtered RF line (a) and corresponding spectrum (b). The filter center frequency was equal to $4 \mathrm{MHz}$. thus were considered to be negligible. The acquired RF data sets were used to calculate the attenuation maps for each angle separately. Then, the attenuation maps were averaged.

Additionally, data was processed by means of frequency compounding (FC) technique. In this case, the band-pass Butterworth filters of fourth order were used. The frequency interval between filters center frequencies was equal to $0.25 \%$ of the filter bandwidth, thus filters bands were overlapping. The use of 12 different filters bandwidths $-[0.5,1,1.5, \ldots, 6] \mathrm{MHz}-$ was tested. The combined bands of the filters covered the closed interval between 1 and $12 \mathrm{MHz}$. For example, for the bandwidth equal to $6 \mathrm{MHz}$ four filters with center frequencies equal to 4, 5.5, 7 and $8.5 \mathrm{MHz}$ were generated. The RF line and corresponding spectra after filtration by the first and fourth filter are presented in Figs. 7 and 8 respectively. The same RF line without filtration is presented in Fig. 5a.

a)

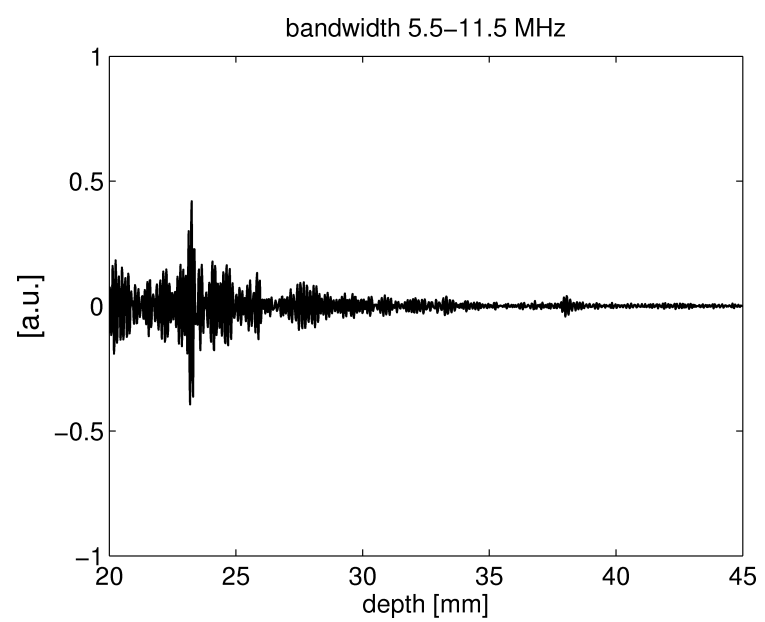

b)

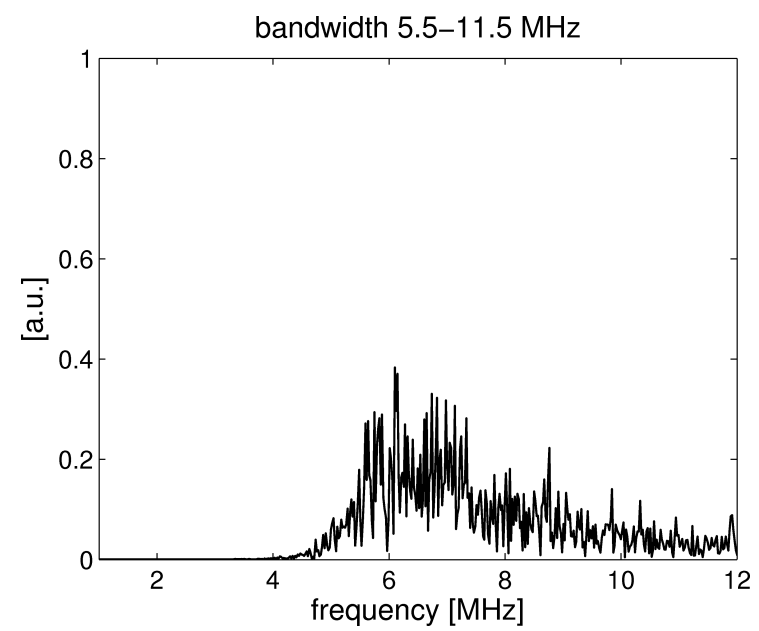

Fig. 8. The filtered RF line (a) and corresponding spectrum (b). The filter center frequency was equal to $8.5 \mathrm{MHz}$. 
The $f_{m}$ lines estimated from all four filtered RF lines are presented in Fig. 9. The energies of the filtered RF signals differ according to filtration (Figs. 7 and 8) which imply their different signal-to-noise ratio (SNR). The compounded frequency $f_{m}$ line (Fig. 9b) is an average of component $f_{m}$ lines (Fig. 9a) weighted by energies of filtered signals. The component $f_{m}$ lines presented in Fig. 9a were averaged with weights equal to $0.29,0.33,0.26$ and 0.12 (from the lowest to the highest). These $f_{m}$ lines correspond with center frequencies of band-pass filters equal to 4, 5.5, 7 and $8.5 \mathrm{MHz}$ respectively. It is worth noting that for filtration with center frequency equal to $8.5 \mathrm{MHz}$ the $f_{m}$ line starts to increase around $35 \mathrm{~mm}$ depth. In this case the energy of the signal is low, which means that SNR is low as well. Moreover, the SNR decreases with depth due to attenuation. Thus, the noise component of the signal starts to dominate at some point. This causes that the $f_{m}$ line starts to rise towards the mean

a)

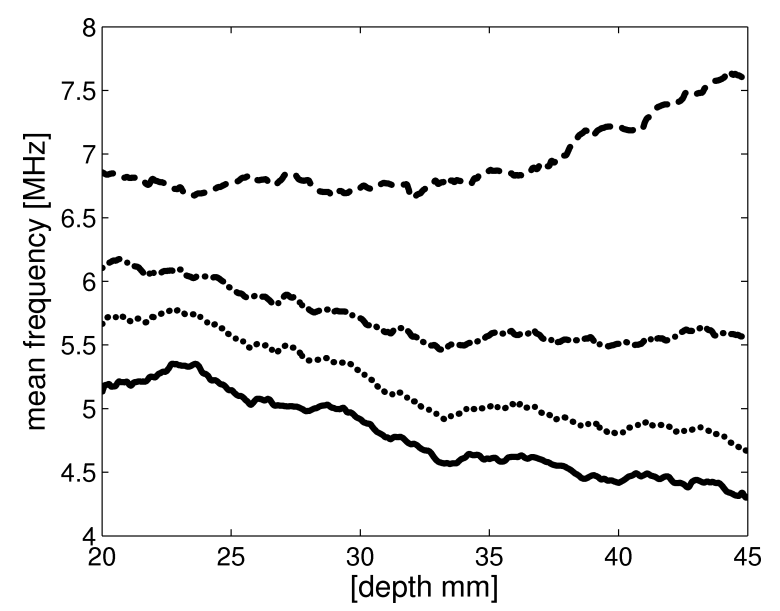

b)

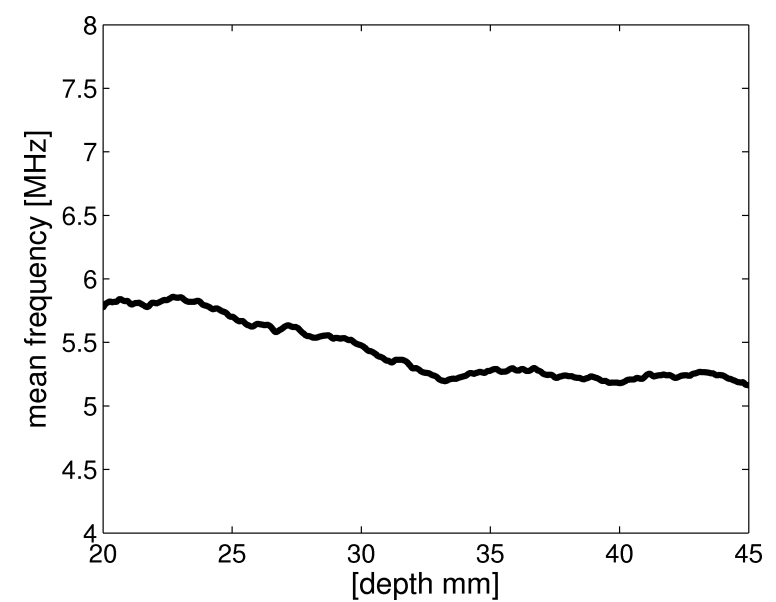

Fig. 9. The component $f_{m}$ lines from filtered RF line (a) and their energy weighted average (b). The successive $f_{m}$ lines starting from the lowest correspond with center frequencies of band-pass filters equal to 4, 5.5, 7 and $8.5 \mathrm{MHz}$ respectively. frequency of the noise while the penetration depth increases. In fact, all of the $f_{m}$ estimates are affected by decreasing SNR which distorts the attenuation estimates. Thus, the energy weighting of the $f_{m}$ lines is used to assure the greater impact on the final estimate for the $f_{m}$ lines with higher SNR. Additional reduction of the effects of decreasing SNR was provided by use of a reference phantom, which is described in next subsection.

\subsection{Compensation of diffraction and noise effects}

The model used to estimate the attenuation (Eq. (8)) does not take into account the diffraction effects as well as the decreasing SNR. In order to compensate these effects a second phantom (1126 B) with uniform attenuation of $0.5 \mathrm{~dB} /(\mathrm{MHz} \cdot \mathrm{cm})$ was used as a reference. Nine RF data sets from the uniform phantom were acquired. Each data set represented different phantom section, that is to say for each acquisition the acoustical beams interacted with different part of the phantom. Next, the RF data sets were processed and the attenuation maps were estimated. Then, the attenuation maps were averaged. In the next step the correction map was calculated as a difference of the averaged attenuation map and the nominal attenuation coefficient value of the phantom $(0.5 \mathrm{~dB} /(\mathrm{MHz} \cdot \mathrm{cm}))$. Such correction map was created for each tested combination of FC parameters depicted in "SC and FC processing" subsection and without the FC processing as well. In other words the processing of reference $\mathrm{RF}$ data sets corresponded with processing of RF data from the phantom containing high attenuating cylinder. Finally, the correction map was used when the attenuation was estimated in inhomogeneous phantom. The diffraction correction was realized by subtracting the correction map from the estimated attenuation map. In Fig. 10 the attenuation estimates are presented

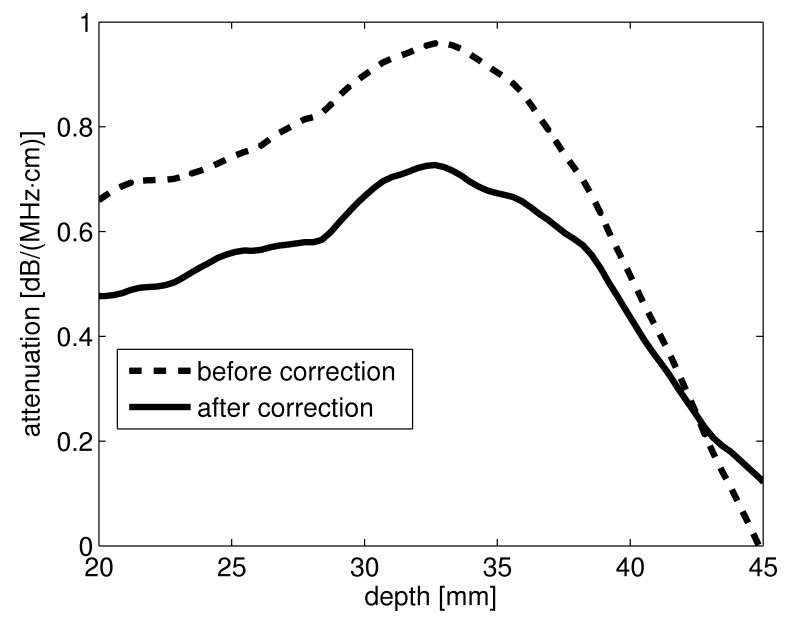

Fig. 10. Attenuation estimates before and after compensation for diffraction effects. 
before and after the compensation for diffraction and decreasing SNR. The lines in Fig. 10 correspond with the lines presented in Fig. 5. The SC and FC processing was not involved in this case.

The data from uniform phantom was also used to estimate the effective spectrum variance (parameter $\sigma_{0}^{2}$ in Eq. (8)) used in the attenuation estimation. The $\sigma_{0}^{2}$ was estimated from the slope of the linear function fitted to the average of all $f_{m}$ lines from all nine measurements.

\section{Results}

In order to reveal the presence of the attenuation inhomogeneity in the tissue phantom, the attenuation imaging technique was applied and the resulting atten- a)

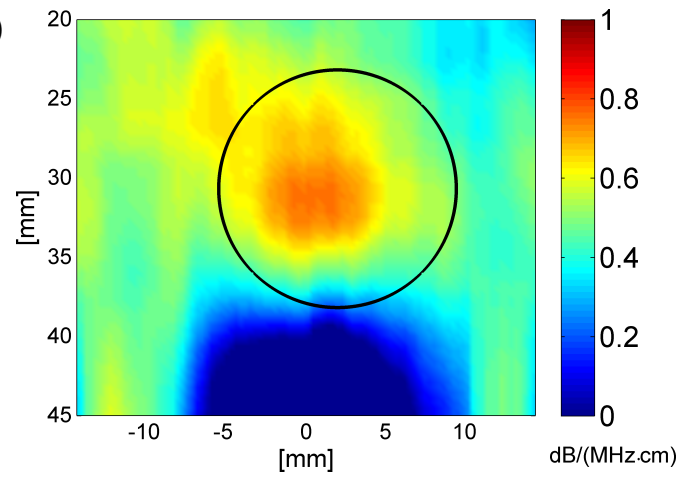

b)

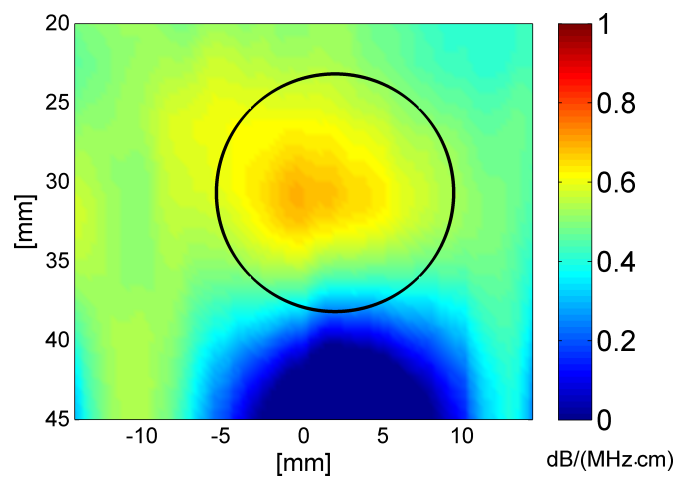

Fig. 11. The attenuation maps of the phantom obtained using $\mathrm{CB}$ (a) and $\mathrm{CB}$ combined with SC technique (b).

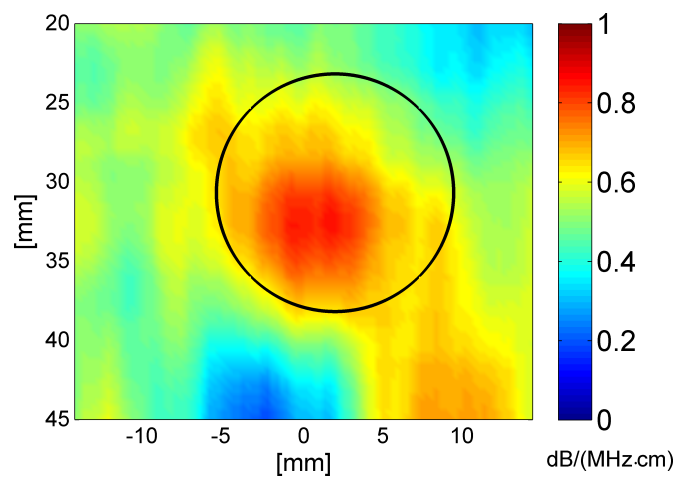

Fig. 12. The attenuation maps of the phantom obtained using $\mathrm{CB}$ combined with FC technique.
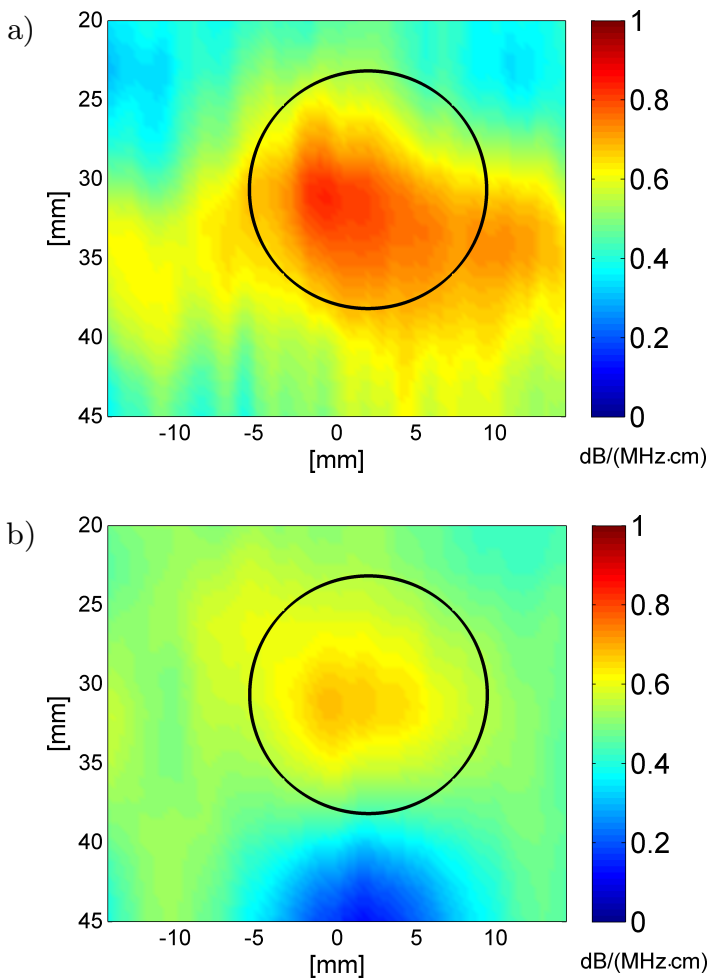

Fig. 13. The attenuation maps obtained using $\mathrm{CB}+\mathrm{FC}+\mathrm{SC}$ techniques for filters bandwidth equal to $0.5 \mathrm{MHz}$ (a) and $6 \mathrm{MHz}(\mathrm{b})$.

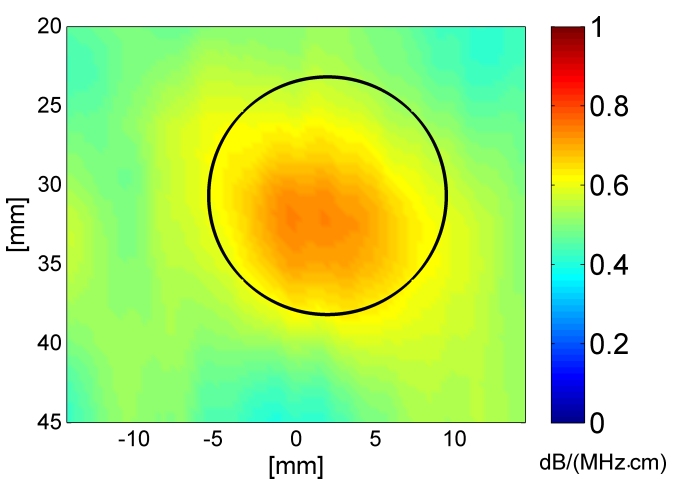

Fig. 14. The attenuation maps obtained using $\mathrm{CB}+\mathrm{FC}+\mathrm{SC}$ techniques for filters bandwidth equal to $2.5 \mathrm{MHz}$.

uation maps are presented in Figs. 11-14. The location of the attenuating cylinder in all of the presented attenuation maps is marked by the black circle. To evaluate the accuracy of attenuation estimates acquired using $\mathrm{CB}, \mathrm{CB}+\mathrm{FC}, \mathrm{CB}+\mathrm{SC}$ and $\mathrm{CB}+\mathrm{FC}+\mathrm{SC}$ techniques, the root-mean-square errors (RMSE) for all attenuation maps were calculated in accordance with Eq. (10)

$$
\operatorname{RMSE}=\sqrt{\frac{\sum_{i=1}^{n}\left(\alpha_{e}(i)-\alpha_{0}(i)\right)^{2}}{n}},
$$

where $\alpha_{e}(i)$ is the attenuation estimate in point $i, \alpha_{0}(i)$ is the nominal attenuation in point $i$ and $n$ is the number of points (pixels) in the attenuation map. 
The attenuation maps obtained with use of the $\mathrm{CB}$ and $\mathrm{CB}+\mathrm{SC}$ are presented in Fig. 11. The highly attenuating cylinder is clearly visible in both cases, however the use of spatial compounding results in a smoother image. The RMSE for $\mathrm{CB}$ and $\mathrm{CB}+\mathrm{SC}$ images were equal to 0.26 and $0.20 \mathrm{~dB} /(\mathrm{MHz} \cdot \mathrm{cm})$ respectively. Therefore, the use of SC technique results with increase in estimation accuracy. There is an artifact beneath the attenuating region visible on both images which probably results from the weaker echo signal in the acoustic shadow. The proposed mechanism of the artifact formation is presented as follows. The propagation through the high attenuating cylinder decreases the signal-to-noise ratio (SNR). When the SNR is low, then the noise component starts to dominate in the mean frequency estimate $\left(f_{m}\right)$. The mean frequency of the noise is approximately constant. Therefore, when the SNR of the received signal decreases, the $f_{m}$ is pulled towards the mean frequency of the noise. Thus, it decreases more slowly or even rises which results in attenuation underestimation.

The attenuation map obtained with use of $\mathrm{CB}+\mathrm{FC}$ with filter bandwidth equal $2.5 \mathrm{MHz}$ is presented in Fig. 12. The use of frequency compounding results in strong reduction of the artifact beneath the high attenuating cylinder. The reduction of the artifact results in smaller RMSE comparing to the $\mathrm{CB}$ and $\mathrm{CB}+\mathrm{SC}$ cases - the RMSE in $\mathrm{CB}+\mathrm{FC}$ case was equal to $0.11 \mathrm{~dB} /(\mathrm{MHz} \cdot \mathrm{cm})$. However the $\mathrm{CB}+\mathrm{SC}$ attenuation map seems to be smoother.

The attenuation images obtained using combined $\mathrm{CB}+\mathrm{FC}+\mathrm{SC}$ techniques are presented in Figs. 13 and 14. Figure 13 presents the results of frequency compounding with non-optimal filters bandwidth. The high attenuating cylinder is visible in both cases, however for too narrow filters (Fig. 13a) the shape is deformed and is not circular. The use of wide filters (Fig. 13b) results in image similar to image obtained using $\mathrm{CB}+\mathrm{SC}$ techniques, however the artifact beneath the cylinder is much smaller. The best image was obtained for filters bandwidth equal to $2.5 \mathrm{MHz}$ (Fig. 14). The high attenuating cylinder is clearly visible, the shape is close to circular and there is no artifact beneath the cylinder.

The RMSE for attenuation maps estimated using $\mathrm{CB}+\mathrm{FC}+\mathrm{SC}$ techniques with different filters bandwidth are presented in Fig. 15. The smallest RMSE $(0.054 \mathrm{~dB} /(\mathrm{MHz} \cdot \mathrm{cm}))$ was obtained using frequency compounding with filters bandwidth equal $2.5 \mathrm{MHz}$. The use of wider or narrower filters results in decreasing of accuracy, however even for the worst cases (filters bandwidth equal to 0.5 or $6 \mathrm{MHz}$ ) the RMSE $(0.096 \mathrm{~dB} /(\mathrm{MHz} \cdot \mathrm{cm}))$ was lower than RMSE of estimate obtained without $\mathrm{FC}$ technique $(0.20 \mathrm{~dB} /(\mathrm{MHz} \cdot \mathrm{cm})-$ Fig. $11 \mathrm{~b})$.

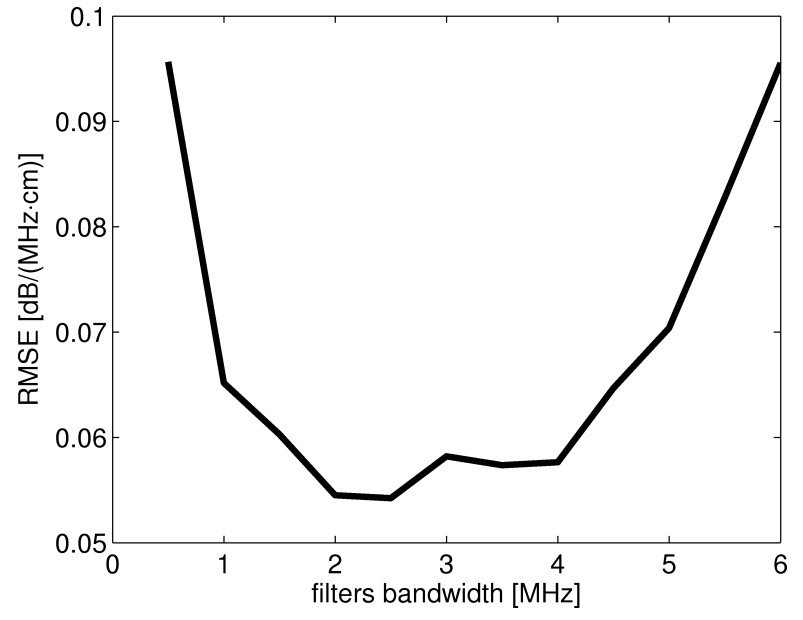

Fig. 15. The RMSE of attenuation maps obtained using $\mathrm{CB}+\mathrm{FC}+\mathrm{SC}$ techniques for different filters bandwidth.

\section{Conclusions}

The attenuation estimation method based on tracking of the spectral mean frequency was presented in this paper. The method was used to reconstruct the attenuation distribution image of the tissue phantom. In order to increase the quality of final images, the raw RF data collected using Classical Beamforming (CB) was processed using Spatial Compounding (SC) and Frequency Compounding (FC) techniques. The attenuation imaging technique revealed the high attenuating cylinder that was almost invisible in the classical B-mode image. The cylinder was detected by all $\mathrm{CB}, \mathrm{CB}+\mathrm{SC}, \mathrm{CB}+\mathrm{FC}$ and $\mathrm{CB}+\mathrm{FC}+\mathrm{SC}$ techniques. The values of RMSE show that the use of separate FC or SC technique improve the accuracy of attenuation maps, but FC is much more effective. The use of $\mathrm{CB}+\mathrm{FC}$ technique results with the $58 \%$ reduction of the RMSE, while for the $\mathrm{CB}+\mathrm{SC}$ technique the reduction is equal to $23 \%$. However, the use of $\mathrm{CB}+\mathrm{FC}+\mathrm{SC}$ with optimal filters bandwidth results in the highest accuracy of attenuation determination in terms of the RMSE and reproduction of the circular shape of the cylinder cross-section The reduction of the RMSE in that case equals $79 \%$. Moreover, the shadow artifact is eliminated in the attenuation image calculated using $\mathrm{CB}+\mathrm{FC}+\mathrm{SC}$ with optimal filters bandwidth. The presented results indicate that the combined spatial and frequency compounding techniques can efficiently improve the quality and accuracy of parametric attenuation images.

\section{Acknowledgments}

This work has been partly supported by the project 2011/01/B/ST7/06728 financed by Polish National Science Centre and project POIG.01.03.01-14-012/08- 
00 co-financed by the European Regional Development Fund under the Innovative Economy Operational Programme. Project carried out with the use of CePT infrastructure within the Innovative Economy Operational Programme for 2007-2013.

\section{References}

1. Bigelow T.A., McFarlin B.L., O'Brien W.D., OELzE M.L. (2008), In vivo ultrasonic attenuation slope estimates for detection cervical ripening in rats: Preliminary results, Journal of Acoustical Society of America, 123, 3, 1794-1800.

2. CobBold R.S.C. (2007), Foundations of Biomedical Ultrasound, Oxford University Press.

3. Evans D.H., McDicken W.N. (2000) Doppler Ultrasound: Physics, Instrumentation and Signal Processing, John Wiley \& Sons Ltd.

4. Golyandina N., Nekrutkin V., Ahigljavsky A. (2001), Analysis of time Series Structure: SSA and related techniques, Chapman \& Hall/CRC.

5. Klimonda Z., Litniewski J., Nowicki A. (2009), Spatial Resolution of Attenuation Imaging, Archives of Acoustics, 34, 4, 461-470.

6. Klimonda Z., Litniewski J., Nowicki A. (2010), Tissue attenuation estimation from backscattered ultrasound using spatial compounding technique - preliminary results, Archives of Acoustics, 35, 4, 643-651.

7. Klimonda Z., Litniewski J., Nowicki A. (2011), Synthetic Aperture technique applied to tissue attenuation imaging, Archives of Acoustics, 36, 4, 927-935.
8. Laugier P., Berger G., Fink M., Perrin J. (1985), Specular reflector noise: effect and correction for in vivo attenuation estimation, Ultras. Imag., 7, 277-292.

9. Lu Z.F., Zagzebski J., Lee F.T. (1999), Ultrasound Backscatter and Attenuation in Human Liver With Diffuse Disease, Ultrasound in Med. \& Biol., 25, 7, 1047-1054.

10. McFarlin B.L., Bigelow T.A., LAYBed Y., O'Brien W.D., Oelze M.L., Abramowicz J.S. (2010), Ultrasonic attenuation estimation of the pregnant cervix: a preliminary results, Ultrasound in Obstetrics and Gynecology, 36, 218-225.

11. Nieminen H.J., SaArakkala S., LaAsanen M.S., Hirvonen J., JuRvelin J.S., TÖyräs J. (2004), Ultrasound Attenuation in Normal and Spontaneously Degenerated Articular Cartilage, Ultrasound in Med. \& Biol., 30, 4, 493-500.

12. Saijo Y., SASAKi H. (1996), High Frequency Acoustic Properties of Tumor Tissue, [in:] Ultrasonic Tissue Characterization, Dunn F., Tanaka M., Ohtsuki S., Saijo Y., 217-230 Springer-VerlagTokio, Hong-Kong.

13. Szabo T.L. (2004), Diagnostic Ultrasound Imaging: Inside Out, Academic Press.

14. Worthington A.E., Sherar M.D. (2001), Changes in UltrasoundProperties of Porcine Kidney Tissue During Heating, Ultrasound in Med. \& Biol., 27, 5, 673-682.

15. Zderic V., Keshavarzi A., Andrew A.M., VAezy S., MARTin R.W. (2004), Attenuation of Porcine Tissues In Vivo After High Intensity Ultrasound Treatment, Ultrasound in Med. \& Biol., 30, 1, 61-66. 